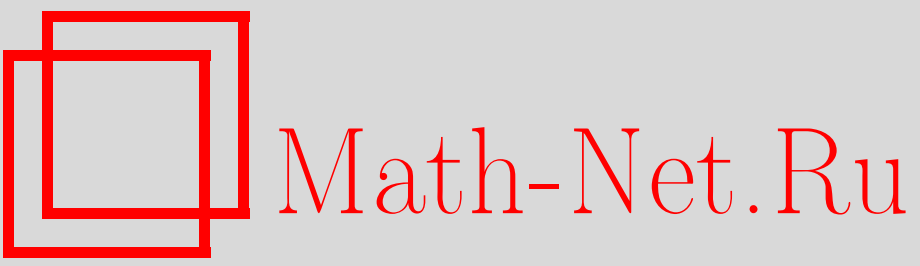

Т. А. Иоанниду, А. Куйрукидис, Н. Д. Влахос, Аналитический подход к $Q$ боллам, ТМФ, 2005, том 144, номер 2, 342-347

DOI: https://doi.org/10.4213/tmf1859

Использование Общероссийского математического портала Math-Net.Ru подразумевает, что вы прочитали и согласны с пользовательским соглашением

http://www . mathnet.ru/rus/agreement

Параметры загрузки:

IP: 52.87 .193 .239

26 апреля 2023 г., 10:08:07 
ТЕОРЕТИЧЕСКАЯ

И МАТЕМАТИЧЕСКАЯ

ФИЗИКА

Том 144, № 2

август, 2005

(C) 2005 г. $\quad$ Т. А. Иоанниду* , А. Куйрукидис ${ }^{\dagger}$, Н. Д. Влахос ${ }^{\dagger}$

\section{АНАЛИТИЧЕСКИЙ ПОДХОД К $Q$-БОЛЛАМ}

Представлен аналитический подход для построения решений вида $Q$-боллов в общем случае потенциала шестого порядка. В частности, показано, что симметризованная функция Вудса-Саксона описывает профиль $Q$-болла, а потому энергию и заряд можно вычислить явно.

Ключевые слова: нетопологические солитоны, заряд Нётер, механический аналог.

\section{1. ВВЕДЕНИЕ}

$Q$-боллы являются когерентными состояниями комплексного скалярного поля, несущими глобальный $U(1)$-заряд, которые можно понимать как связанные состояния скалярных частиц, появляющиеся в виде устойчивых классических решений с вращаюшейся зависящей от времени внутренней фазой [1], [2]. Они характеризуются сохраняющимся нетопологическим зарядом $Q$ (зарядом Нётер), что обеспечивает их сушествование и устойчивость [3]. В действительности $Q$-боллы естественным образом возникают в суперсимметричных теориях [4], и их устойчивость имеет значение для космологии, поскольку если устойчивые $Q$-боллы формируются в ранней Вселенной, то они могут давать вклад в темную материю [5].

Хотя $Q$-боллы сушествуют в разнообразных моделях теории поля, мы рассмотрим лагранжиан $U(1)$ модели Голдстоуна

$$
\mathcal{L}=\frac{1}{2} \partial_{\mu} \phi \partial^{\mu} \bar{\phi}-U(|\phi|),
$$

где $\phi$ - комплексное скалярное поле в трех пространственных измерениях, а потенциал $U(|\phi|)$ имеет единственный минимум при $\phi=0$. Эквивалентным образом можно сказать, что имеется сектор скалярных частищ (мезонов) с массой $\sqrt{U^{\prime \prime}(0) / 2}$, несуших $U(1)$ заряд. Соответствуюший функционал энергии имеет вид

$$
E=\int\left(\frac{1}{2}|\dot{\phi}|^{2}+\frac{1}{2}|\nabla \phi|^{2}+U(|\phi|)\right) d^{3} x,
$$

* Mathematics Division, School of Technology, Aristotle University of Thessaloniki, Thessaloniki 54124, Greece. E-mail: ti3@gen.auth.gr

${ }^{\dagger}$ Physics Department, Aristotle University of Thessaloniki, Thessaloniki 54124, Greece. E-mail: kouirouki@astro.auth.gr, vlachos@physics.auth.gr 
а сохраняющийся (из-за глобальной $U(1)$-симметрии) ток Нётер есть

$$
J_{\mu}=\frac{1}{2 i}\left(\bar{\phi} \partial_{\mu} \phi-\phi \partial_{\mu} \bar{\phi}\right)
$$

причем заряд равен

$$
Q=\frac{1}{2 i} \int\left(\bar{\phi} \partial_{t} \phi-\phi \partial_{t} \bar{\phi}\right) d^{3} x
$$

Стационарное решение вида $Q$-болла можно получить, полагая

$$
\phi=e^{i \omega t} f(r)
$$

где $f(r)$ - вешественная радиальная функция профиля, удовлетворяющая обыкновенному дифференциальному уравнению

$$
\frac{d^{2} f}{d r^{2}}+\frac{2}{r} \frac{d f}{d r}=-\omega^{2} f+U^{\prime}(f)
$$

с граничными условиями $f(\infty)=0$ и $f^{\prime}(0)=0$. В каждом случае эффективный потенциал определяется как $U_{\mathrm{eff}}(f)=\omega^{2} f^{2} / 2-U(f)$, а сушествование решений вида $Q$-болла приводит к ограничениям на потенциал $U(f)$ и частоту $\omega: 1)$ эффективная масса решения $f$ должна быть отрицательной, откуда, полагая $U(0)=U^{\prime}(0)=0$ и $U^{\prime \prime}(0)=$ $\omega_{+}^{2}>0$, можно вьвести, что $\left.\omega<\omega_{+} ; 2\right)$ минимум функции $U(f) / f^{2}$ должен достигаться при некотором положительном значении $f$ (скажем, $0<f_{0}<\infty$ ), и сушествование решения требует, чтобы $\omega>\omega_{-}$, где $\omega_{-}^{2}=2 U\left(f_{0}\right) / f_{0}^{2}$. Поэтому $Q$-боллы сушествуют при всех $\omega$ в интервале $\omega_{-}<|\omega|<\omega_{+}$.

Заряд (4) и энергия (2) стационарного решения вида $Q$-болла (5) принимают простой вид:

$$
\begin{aligned}
& Q=4 \pi \omega \int r^{2} f^{2}(r) d r \\
& E=\frac{1}{2} \omega^{2} Q^{2}+4 \pi \int\left(\frac{f^{\prime 2}}{2}+U(f)\right) r^{2} d r .
\end{aligned}
$$

Выбор потенциала при этом неоднозначен, поскольку единственное требование состоит в том, чтобы отношение $U(f) / f^{2}$ имело локальньй минимум при некотором ненулевом значении $f$. Однако мы рассмотрим случай, когда

$$
U(f)=\frac{m_{1}^{2}}{2} f^{2}-\lambda_{1} f^{4}+\mu_{1} f^{6}
$$

при котором $\omega_{+}=m_{1}$ и $\omega_{-}=\sqrt{\left(2 m_{1}^{2} \mu_{1}-\lambda_{1}^{2}\right) /\left(2 \mu_{1}\right)}$. Устойчивые $Q$-боллы сушествуют при $\sqrt{\left(2 m_{1}^{2} \mu_{1}-\lambda^{2}\right) /\left(2 \mu_{1}\right)}<\omega<m_{1}$. Тогда уравнение движения (6) принимает вид

$$
\frac{d^{2} f}{d r^{2}}+\frac{2}{r} \frac{d f}{d r}=\alpha^{2} f-4 \lambda_{1} f^{3}+6 \mu_{1} f^{5}
$$


где $\alpha^{2}=m_{1}^{2}-\omega^{2}$. Если интерпретировать $f$ как положение частишы, а $r$ как время, то уравнение (10) можно воспринимать как ньютоновское уравнение движения частицы единичной массы, подчиненной вязкому трению и движущейся в эффективном потенциале $\omega^{2} f^{2} / 2-U(f)$. Это приводит к ограничениям на потенциал и частоту $\omega$, обеспечиваюшим сушествование решения вида $Q$-болла (см. обсуждение в разделе 2 ). По поводу дальнейших подробностей мы отсылаем читателя к работе Коулмена [1], где доказана фундаментальная теорема сушествования для этих $Q$-боллов и найдены их низшие возбуждения.

\section{2. АСИМПТОТИЧЕСКИЕ ПРЕДЕЛЫ ПОТЕНЦИАЛА}

С точки зрения механики решение вида $Q$-болла описывает движение частицы с трением в потенциале

$$
U_{\text {eff }}(f)=-\frac{1}{2} \alpha^{2} f^{2}+\lambda_{1} f^{4}-\mu_{1} f^{6}, \quad \lambda_{1}, \mu_{1}>0 .
$$

Вьполняя масштабное преобразование $r \rightarrow r / \alpha$ и полагая $f(r)=f(0) \psi(a r)$, где $\psi(0)=1$, преобразуем уравнение (10) к виду

$$
\frac{d^{2} \psi}{d r^{2}}+\frac{2}{r} \frac{d \psi}{d r}=\psi-4 \lambda \psi^{3}+6 \mu \psi^{5}
$$

где $\lambda=\lambda_{1} f(0)^{2} / \alpha^{2}$ и $\mu=\mu_{1} f(0)^{4} / \alpha^{2}$, а эффективный потенциал (11) принимает вид

$$
U(\psi)=-\frac{1}{2} \psi^{2}+\lambda \psi^{4}-\mu \psi^{6}
$$

Интегрируя уравнение (12), получаем

$$
\int_{0}^{\infty} r^{n-1}\left(\frac{d \psi}{d r}\right)^{2} d r=\frac{2 n}{4-n} \int_{0}^{\infty} r^{n-1}\left(-\frac{1}{2} \psi^{2}+\lambda \psi^{4}-\mu \psi^{6}\right) d r, \quad n \neq 0
$$

Специальные случаи $n=3$ и $n=0$ приводят соответственно к условиям

$$
\begin{aligned}
\frac{1}{2} \int_{0}^{\infty} r^{2}\left(\frac{d \psi}{d r}\right)^{2} d r & =3 \int_{0}^{\infty} r^{2}\left(-\frac{1}{2} \psi^{2}+\lambda \psi^{4}-\mu \psi^{6}\right) d r \\
2 \int_{0}^{\infty} r^{-1}\left(\frac{d \psi}{d r}\right)^{2} d r & =-\frac{1}{2} \psi(0)^{2}+\lambda \psi(0)^{4}-\mu \psi(0)^{6} \equiv U(1) .
\end{aligned}
$$

Первое условие является теоремой Деррика (или вириальной теоремой): в трехмерной модели кинетическая энергия в три раза превосходит потенциальную; второе же условие описывает диссипацию энергии в механической системе.

При рассмотрении механического аналога задачи на $U(1)$ налагаются определенные ограничения. Для частицы, исходно расположенной в точке $\psi(0)=1$, начинающей соскальзывать с потенциальной стенки и в конце концов останавливающейся в точке 
$\psi(\infty)=0$, потенциальная энергия $(13)$ в начале координат $U(1)$ должна быть поглощена за счет члена с трением $(2 / r) d \psi / d r$ (здесь $r$ соответствует временно́й переменной). Поэтому ограничения на $U(1)$ должны быть как снизу, так и сверху, поскольку при больших $U(1)$ частица перелетит через верхнюю точку, а при малых $U(1)$ она ее не достигнет.

Исходная потенциальная энергия $U(1)$ должна быть положительной, $\mu \leqslant \lambda-1 / 2$; $U^{\prime}(1)$ также должна быть положительной (притягивающая “сила"): $\mu \leqslant(4 \lambda-1) / 6$. Эти условия выполнены при $1 / 2<\lambda<1$ и $\lambda>1$, соответственно. В зависимости от формы потенциала (т.е. от конкретных значений $\lambda$ и $\mu$ ) возможны следуюшие отличные друг от друга случаи.

I. Приближение тонких стенок. В этом случае $\psi(0)$ лежит вблизи максимума эффективного потенциала, являюшегося глубоким. Тогда $U^{\prime}(1)=-1+4 \lambda-6 \mu \approx 0$ должна быть положительной и близкой к нулю (медленное скатывание), a $U^{\prime \prime}(1)$ - отрицательной (выпуклая область) и иметь порядок единицы. Решение вида $Q$-боллаприближенно лежит на линии

$$
\mu=\frac{1}{6}(4 \lambda-1),
$$

и исходная потенциальная энергия линейно зависит от $\lambda$ :

$$
U(1)=\frac{1}{3}(\lambda-1), \quad \lambda>1 .
$$

II. Приближение толстых стенок. Здесь потенциал является мелким и максимумы расположены высоко, $U^{\prime \prime}(1)$ является положительной и большой (вогнутая область) и $\mu$ мало. Исходная потенциальная энергия $U(1)$ возрастает с $\lambda$ и достигает своего максимального значения при $\mu \rightarrow 0$. Это значение можно определить численно, и результат равен $\lambda_{\max } \simeq 4.70137$. Однако реальная функциональная зависимость $U(1)$ от $\lambda$ не известна, и ее следует определить.

В предположении степенно́го поведения потенциала в главном порядке вида

$$
U(1)=\kappa \lambda^{n}
$$

из уравнения (13) следует, что решение вида $Q$-болла лежит на линии

$$
\mu=-\frac{1}{2}+\lambda-\kappa \lambda^{n}
$$

которая определяет $\kappa$ через $\lambda_{\max }\left(\right.$ поскольку $\left.\mu\left(\lambda_{\max }\right)=0\right)$ :

$$
\kappa=\frac{2 \lambda_{\max }-1}{2 \lambda_{\max }^{n}} .
$$

При этом значение $n$ в формуле (19) определяется исходя из требования, чтобы переход из области толстых стенок в область тонких был гладким, откуда, как доказано в работе [6], следует $n=3$, а потому $\kappa=1 / 24.75$. 


\section{3. СВОЙСТВА $Q$-БОллОВ}

Используя формулы масштабного преобразования из предыдушего раздела, можно аналитически определить зависимость функций $E, Q$ и $f(0)$ от $\alpha$. Исходное значение поля $f(0)$ в терминах преобразованных формул есть

$$
f(0)=\alpha \sqrt{\frac{\lambda}{\lambda_{1}}}, \quad \alpha=\sqrt{\frac{\mu}{\mu_{1}}} \frac{\lambda_{1}}{\lambda},
$$

а соответствуюшие функционалы заряда (7) и энергии (8) принимают вид

$$
\begin{aligned}
& Q=\frac{4 \pi \omega}{\alpha} \frac{\lambda}{\lambda_{1}} \int_{0}^{\infty} \psi^{2} r^{2} d r \\
& E=\frac{\alpha^{2}+2 \omega^{2}}{2 \omega} Q+4 \pi \alpha \frac{\lambda}{\lambda_{1}} \int_{0}^{\infty}\left(\frac{1}{2} \psi^{\prime 2}-\lambda \psi^{4}+\mu \psi^{6}\right) r^{2} d r .
\end{aligned}
$$

В работе [7] было показано, что пробная функция, удовлетворяющая граничным условиям и имеюшая правильное асимптотическое поведение, является симметризованным профилем Вудса-Саксона:

$$
\phi(r)=\frac{c}{\sqrt{1+c_{1} \operatorname{ch}(b r)}},
$$

в терминах которого функционалы заряда (23) и энергии (24) можно явно вычислить как

$$
\begin{aligned}
Q= & \frac{4 \pi \omega}{\alpha} \frac{\lambda}{\lambda_{1}} \frac{c^{2}}{3 b^{3}} i_{0} \\
E= & 4 \pi \frac{c^{2}}{3 b^{3}} \sqrt{\frac{\mu}{\mu_{1}}}\left\{\left(\frac{\left(m^{2}+\omega^{2}\right)}{2 \alpha} \frac{\lambda}{\lambda_{1}} \sqrt{\frac{\mu_{1}}{\mu}}+\mu c^{4}-\lambda c^{2}-\frac{1}{8} b^{2} c_{1}^{2}\right) i_{0}+\right. \\
& \left.+\left(2 \mu c^{4}-\lambda c^{2}-\frac{1}{4} b^{2} c_{1}^{2}\right) c_{1} \frac{d i_{0}}{d c_{1}}+\frac{1}{2}\left(\mu c^{4}+\frac{1}{8} b^{2}\left(1-c_{1}^{2}\right)\right) c_{1}^{2} \frac{d^{2} i_{0}}{d c_{1}^{2}}\right\} .
\end{aligned}
$$

Здесь $i_{0}$ - функция от $c_{1}$, задаваемая в виде

$$
i_{0}\left(c_{1}\right)=\frac{1}{\sqrt{1-c_{1}^{2}}} \operatorname{arcch}\left(\frac{1}{c_{1}}\right)\left[\pi^{2}+\operatorname{arcch}\left(\frac{1}{c_{1}}\right)^{2}\right], \quad c_{1}<1 .
$$

Заметим, что $\lambda_{1}, \mu_{1}, m$ - фиксированные внешние параметры, $\lambda$ изменяется непрерывно от 1 до 4.70137, а $\mu$ - известная функция от $\lambda$, заданная одной из формул (17) или (20) в зависимости от режима. Поэтому имеется только два неопределенных параметра параметры профиля $b$ и $c_{1}$.

Эти параметры можно определить, налагая условия (15) и (16) на $\phi(r)$, т.е.

$$
\begin{gathered}
\frac{1}{2} b^{2} c_{1}^{2} c^{2} \int_{0}^{\infty} \frac{d r}{r} \frac{\operatorname{sh}^{2} r}{\left(1+c_{1} \operatorname{ch} r\right)^{3}}=U(1) \\
\left(\frac{1}{2}-\lambda c^{2}+\mu c^{4}-\frac{b^{2} c_{1}^{2}}{24}\right) i_{0}+\left(2 \mu c^{4}-\frac{b^{2} c_{1}^{2}}{12}-\lambda c^{2}\right) c_{1} \frac{d i_{0}}{d c_{1}}+ \\
+\frac{c_{1}^{2}}{2}\left(\mu c^{4}+\frac{b^{2}}{24}\left(1-c_{1}^{2}\right)\right) \frac{d^{2} i_{0}}{d c_{1}^{2}}=0 .
\end{gathered}
$$


Заметим, что получаемые таким образом значения $c_{1}$ и $b$ являются универсальными, поскольку они не зависят от геометрических параметров потенциала. Тем не менее значения энергии и заряда зависят от конкретного вида потенциала, т.е. от значений $\lambda_{1}, \mu_{1}$ и $m$. Эти значения стремятся к бесконечности в каждом из пределов, поскольку в пределе тонких стенок $c_{1} \rightarrow 0$, а в пределе толстых стенок $\alpha \rightarrow 0$. Интересно подчеркнуть, что область применимости приближения толстых стенок очень широка и дает удовлетворительные результаты даже в области тонких стенок. Представляется, что в этом классе теорий $Q$-боллы предпочитают быть “толстыми”.

\section{4. ЗАКЛЮЧЕНИЕ}

Мы универсальным способом исследовали феноменологически сушественные свойства $Q$-боллов. В частности, было показано, что, используя геометрические характеристики скалярного потенциала, можно вычислить как исходное значение поля, которое приводит к решению вида $Q$-болла, так и соответствуюшие заряд и энергию солитона. Мы нашли, что эта схема приводит к удовлетворительным результатам во всей области изменения параметров, так что нет необходимости полагаться на приближения типа тонких или толстых стенок. Энергию и заряд солитона можно далее вычислить аналитически и сравнить с результатами численных вычислений из работы [8].

Мы полагаем, что подобную же аргументацию можно применить к изучению функции профиля зависимости энергия-заряд в потенциалах всех типов.

\section{Список литературы}

[1] S. Coleman. Nucl. Phys. B. 1985. V. 262. P. 263.

[2] T. D. Lee. Particles Physics and Introduction to Field Theory. London: Harwood, 1981.

[3] J.K. Drohm, L. P. Yok, Y. A. Simonov, J.A. Tyon, V.I. Veselov. Phys. Lett. B. 1981. V. 101. Р. 204; Т.И. Белова, А.Е. Кудрявцев. ЖЭТФ. 1989. Т. 95. С. 13; D. К. Hоng. J. Low Temp. Phys. B. 1998. V. 71. P. 483.

[4] A. Kusenko. Phys. Lett. B. 1997. V. 405. P. 108.

[5] A. Kusenko, M. Shaposhnikov. Phys. Lett. B. 1998. V. 418. P. 46; K. Enqvist, J. McDonald. Nucl. Phys. B. 1999. V. 538. P. 321

[6] T. Ioannidou, A. Kouiroukidis, N.D. Vlachos. Universality in a class of $Q$-balls solutions: an analytic approach. hep-th/0405209.

[7] T. Ioannidou, N. D. Vlachos. J. Math. Phys. 2003. V. 44. P. 3562.

[8] T. Ioannidou, V. Kopeliovich, N. D. Vlachos. Nucl. Phys. B. 2003. V. 660. P. 156. 